\title{
Molecular and clinical characteristics of $\mathrm{MSH6}$ germline variants detected in colorectal cancer patients
}

\author{
HIROKO TERUI $^{1,4}$, TETSUHIKO TACHIKAWA ${ }^{1}$, MIHO KAKUTA ${ }^{1}$, YOJI NISHIMURA ${ }^{2}$, \\ TOSHIMASA YATSUOKA $^{2}$, KENSEI YAMAGUCHI $^{3}$, KEI YURA $^{4,5}$ and KIWAMU AKAGI ${ }^{1}$ \\ ${ }^{1}$ Division of Molecular Diagnosis and Cancer Prevention, ${ }^{2}$ Gastroenterological Surgery and ${ }^{3}$ Gastroenterology, \\ Saitama Cancer Center, Saitama 362-0806; ${ }^{4}$ The Graduate School of Humanities and Sciences, Ochanomizu University, \\ Tokyo 112-8610; ${ }^{5}$ Center for Informational Biology, Ochanomizu University, Tokyo 112-8610, Japan
}

Received July 9, 2013; Accepted August 16, 2013

DOI: $10.3892 /$ or.2013.2781

\begin{abstract}
The MSH6 gene is one of the mismatch repair genes involved in Lynch syndrome and its mutations account for $10-20 \%$ of Lynch syndrome. Although previous studies suggested that the difference of the geographical region affects the clinical phenotype of Lynch syndrome, there has been no report on the detailed features of Japanese Lynch syndrome patients carrying an MSH6 mutation. The aim of the present study was to investigate the clinical and molecular features of MSH6 mutation carriers in Japan. Surgically resected 1720 colorectal carcinoma specimens were screened by microsatellite instability (MSI) testing and the MSI-high cases were subjected to a germline mutation analysis of the mismatch repair genes $M L H 1, M S H 2$ and $M S H 6$. We investigated the clinical and molecular features of the MSH6 variants, such as the family cancer history, pathological findings, immunohistochemistry, methylation status of the $M L H 1$ promoter and $B R A F$ mutation in the colorectal tumor. Furthermore, the impact of the missense variants on MSH6 protein was predicted by using in silico tools. We identified nine novel pathogenic mutations and eight unclassified missense variants. Among the eight missense variants, three were suspected pathogenic by in silico analysis. We also found that most colorectal cancers in the MSH6 mutation carrier were diagnosed after the age of 50
\end{abstract}

Correspondence to: Dr Kiwamu Akagi, Division of Molecular Diagnosis and Cancer Prevention, Saitama Cancer Center, 818 Komuro Ina, Kita-adachigun, Saitama 362-0806, Japan

E-mail: akagi@cancer-c.pref.saitama.jp

Abbreviations: CRC, colorectal cancer; IHC, immunohistochemistry; LS, Lynch syndrome; MLPA: multiplex ligation- dependent probe amplification; MMR, mismatch repair; MSI, microsatellite instability; MSI-H, high-level of microsatellite instability; MSI-L, low-level of microsatellite instability; MSS, microsatellite stable; VUS, variants of uncertain significance

Key words: Lynch syndrome, MSH6, microsatellite instability, immunohistochemistry, HNPCC, colorectal cancer, endometrial cancer and were localized distally. Furthermore, the mean age at diagnosis of endometrial cancer in Japanese MSH6 mutation carriers (49.2 years) was earlier than previous reports from Western countries (56.5 years). These results may improve the surveillance program for Japanese MSH6 mutation carriers.

\section{Introduction}

Lynch syndrome (LS) is the most common hereditary colorectal cancer syndrome (1), and is an autosomal dominant disease that accounts for $1-5 \%$ of all colorectal cancer (CRC) patients (2-4). LS is associated with germline mutations in one of the DNA mismatch repair (MMR) genes, such as $M L H 1$, MSH2, MSH6 or PMS2 (MIM\#s 120436, 609309, 600678, 600259 , respectively). Carriers of MMR gene mutations are at high risk of developing LS associated cancer in the colorectum, endometrium, small bowel, stomach, ovary, ureter, biliary tract, brain and skin. Individuals at high-risk can be identified by germline genetic testing.

To identify individuals at risk for LS, the Amsterdam criteria and the Bethesda guidelines based on the family cancer history and age at cancer diagnosis have been proposed $(5,6)$. However, MSH6 mutation carriers do not necessarily satisfy these criteria, as they tend to develop CRC at an older age than $M L H 1$ or $M S H 2$ mutation carriers and have reduced penetrance (7-10). Thus, there are some difficulties associated with using these criteria in the clinical practice, and a molecular analysis of the tumor may help to identify individuals who have LS. The tumors from LS patients are characterized by the microsatellite instability (MSI) and a loss of expression of MMR proteins detected by immunohistochemistry (IHC). Routine MSI and IHC testing for CRC patients is an effective screening tool to identify overlooked cases of LS. Approximately 6-15\% of all CRCs display high-level of MSI (MSI-H) and in the majority of these cases, the MMR defects result from inactivation of $M L H 1$ by the methylation of its promoter (11-13). The presence of $M L H 1$ promoter hypermethylation generally suggests sporadic CRC, although there are some exceptions. In addition, the BRAF mutation, p.V600E, has also been identified in colorectal tumors showing MMR deficiency associated with the epigenetic silencing of the MLH1 gene, and previous studies showed that tumors from 
patients with germline mutations in the MMR genes did not show somatic mutations in $B R A F$ (14). Thus, the results of the analyses of MSI, IHC, $M L H 1$ methylation and $B R A F$ mutation in tumors may aid in the diagnosis of LS.

In the present study, we identified 15 novel variants of MSH6 and evaluated the molecular and clinical characteristics of these patients. This is the first report to describe the detailed data of Japanese LS due to MSH6 mutation.

\section{Materials and methods}

Samples and DNA extraction. The CRC samples were collected from patients who were treated at Saitama Cancer Center beginning in 1998, and 1720 paired surgical specimens of primary CRC and normal mucosa were used for the present study. Informed consent was obtained from each patient. Fresh tissue samples were immediately frozen at $-80^{\circ} \mathrm{C}$. Genomic DNA was extracted using the standard method (15). This research protocol was approved by the Institutional Review Board of Saitama Cancer Center.

Clinical database collection. The clinical features, such as the personal and family cancer history and pathological findings of the tumors, were collected from medical records or directly from patients who were provided genetic counseling. The personal and family cancer history was classified into five categories based on the clinical criteria (Table I). The criteria were defined based on both the Amsterdam criteria II (16) and revised Bethesda guidelines (17).

Analysis of microsatellite instability. The MSI status of the colorectal cancers was investigated according to a previously reported method (18). A reference panel of five MSI markers recommended by the National Cancer Institute workshop: BAT-25, BAT-26, D2S123, D5S346 and D17S250, were used. $A$ variation in the number and size of peaks of a marker in tumor DNA compared with that of normal DNA was interpreted to indicate instability for the markers. Tumors were classified as MSI-H if at least two of the five markers showed instability compared with normal tissues, and were classified as low-level of MSI (MSI-L) if one marker showed instability. Tumors showing no instability were classified as microsatellite stable (MSS).

Methylation of the MLH1 promoter and BRAF mutation. The methylation status of the $M L H 1$ promoter in MSI-H CRC was investigated by methylation-specific PCR. In addition, a mutational analysis of $B R A F$ was performed by PCR-RFLP and sequencing and the analysis focused specifically on the V600E mutation. These analyses were carried out by the methods previously reported (19).

Mutation analysis of the MMR genes. Germline mutations in $M L H 1, M S H 2$ and $M S H 6$ were investigated in patients with MSI-H CRC. The mutation analyses were performed by direct sequencing of the entire coding region and large deletions/duplications in the $M L H 1$ and $M S H 2$ genes were also investigated by multiplex ligation-dependent probe amplification (MLPA). The mutation description is according to the reference sequence: NM_000249.2,NM_000251.1 and
NM_000179.2. The G39E variant in MSH6 (rs1042821) was considered to be non-pathogenic due to the high frequency of the allele (20) and the variant was excluded from this report. If a pathogenic mutation was detected in the CRC patients, their relatives were referred to for genetic testing. Informed consent was obtained from each relative before blood samples were collected. The mutation analyses were performed by limiting it to the probands' mutation site.

Immunohistochemical analysis for the MMR protein. Immunohistochemical staining was performed for the MLH1, MSH2, MSH6 and PMS2 proteins. A total of 70 tumor specimens showing MSI-H were tested. The tumor and normal frozen samples were dissected in $4-\mu \mathrm{m}$ serial sections. Frozen sections were fixed with $4 \%$ paraformaldehyde containing $0.5 \%$ triton. Mouse anti-human monoclonal antibodies against MLH1 (G168-728; Cell Marque, Rocklin, CA, USA, at 1:500 dilution), MSH2 (NA27; Calbiochem, Billerica, MA, USA, at 1:500 dilution) and PMS2 (PDM171; Diagnostic BioSystems, Pleasanton, CA, USA, at no dilution) and a rabbit anti-human monoclonal antibody against MSH6 (ab92471; Cambridge, UK, at 1:100 dilution) were used for the present study. The incubation periods were $45 \mathrm{~min}$ at room temperature for MLH1 and MSH2, $2 \mathrm{~h}$ at room temperature for MSH6 and overnight at $4^{\circ} \mathrm{C}$ for PMS2. The reviewers of the immunostaining samples were blinded to the results of the genetic testing. Stained samples were classified into positive (normal expression of nuclear staining) or negative (loss of nuclear staining) groups. It is known that loss of only the MSH6 or PMS2 protein expression is associated with the respective genetic mutations, but that loss of the $\mathrm{MSH} 2$ protein expression is accompanied by loss of MSH6, and loss of MLH1 is accompanied by loss of PMS2 (21).

In silico analysis. In order to classify the MSH6 missense variants into pathogenic or non-pathogenic, the clinical and molecular data and in silico predictions were integrated. The in silico tools, CoDP (22), SIFT (23), PolyPhen-2 (24) and PON-MMR (25) were used in the present study. PolyPhen-2 calculates the values of both HumDiv and HumVar. The HumDiv is used for the diagnosis of Mendelian diseases, and HumVar is used for the evaluation of rare alleles potentially involved in complex phenotypes (24). Both values were used in the present study.

Statistical analysis. The data were calculated as totals, means, medians or percentages. Student's t-test, the Mann-Whitney U-test, one-way analysis of variance (ANOVA) or the KruskalWallis test was used for statistical analyses as appropriate. The analyses were carried out using the PASW Statistics 18.0.0 software program (SPSS, Inc., Chicago, IL, USA), with a value of $\mathrm{P}<0.05$ considered to indicate a statistically significant result.

\section{Results}

Variant analysis. We identified nine pathogenic variants which were truncating mutations and eight missense variants in MSH6. The pathogenic germline mutations were c.619G>T; p.E207X, c.1088delC, c.1149delG, c.1571dupA, c.1909_1913delCTCCT, c.2773G>T; p.Q925X, c.2932C>T; p.Q978X, c.3261dupC and 
Table I. Definition of the clinical criteria.

Criteria

A2 Amsterdam criteria II: three or more family members affected with Lynch syndrome-associated cancer ${ }^{\mathrm{a}}$, one of whom was a first-degree relative of the other two, at least two successive generations were affected and at least one cancer was diagnosed at the age of $<50$. Familial adenomatous polyposis (FAP) was excluded.

B1 Revised Bethesda guidelines 1: a patient who was diagnosed with colorectal cancer at the age of $<50$.

B2 Revised Bethesda guidelines 2: a patient with synchronous or metachronous colorectal, or other Lynch syndrome-related tumor $^{\mathrm{b}}$, regardless of the age at diagnosis.

B4 Revised Bethesda guidelines 4: a patient with colorectal cancer who has one or more first-degree relatives with a Lynch syndrome-related tumor ${ }^{\mathrm{b}}$ diagnosed at the age of $<50$.

B5 Revised Bethesda guidelines 5: a patient with colorectal cancer who has two or more first- or second-degree relatives with Lynch syndrome-related tumor ${ }^{\mathrm{b}}$ regardless of the age at diagnosis.

${ }^{\mathrm{a}}$ Colorectal, endometrial, small bowel, renal pelvis and ureter. ${ }^{\mathrm{b}}$ Colorectal, endometrial, stomach, ovarian, pancreas, ureter, renal pelvis, biliary tract, and brain tumor, sebaceous gland adenomas and keratoacanthomas and carcinoma of the small bowel.

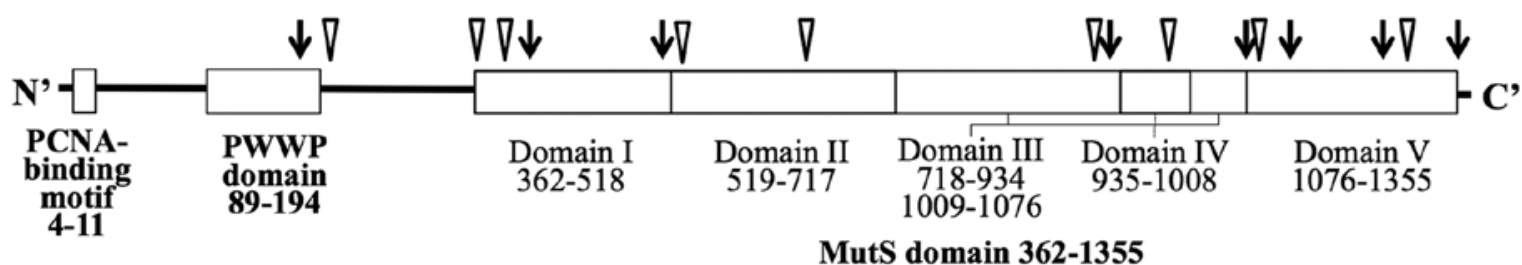

$\nabla$ : Nonsense mutation or frameshift mutation : Missense variant

Figure 1. The distribution of the truncating mutations and missense variants. MSH6 protein is depicted by box diagram. A box indicates a domain structure and a line connecting the boxes indicates an inter-domain sequence. The range of the domain is shown beneath the box. Triangle ( $\Delta$ ) indicates the location of a truncating mutation, i.e. nonsense mutation and frameshift mutation, and directing arrow (l) indicates the location of missense variants. The majority of the mutations were located in the MutS domain, but no spatial preference of the mutation site in the domain was found.

c.3961A >T; p.R1321X. The missense variants were c.532C >T; p.R178C, c.1190A > G (rs63750065); p.Y397C, c.1505T>C; p.I502T, c.2780T >C; p.I927T, c.3244C >T (rs186240214); p.P1082S, c.3464A>G; p.Q1155R, c.3947G>A; p.G1316E and c.4071T $>$ G; p.I1357M (Table II) and these are all variants of uncertain significance (VUS). These variant carriers did not have other mutations in $M L H 1$ and $M S H 2$ except p.R178C variant carrier. None of the nine pathogenic mutations are listed in the InSiGH (17), MMRUV (26) or HGMD (27) databases, and hence, these are novel mutations in MSH6 associated with LS. Other than p.Y397C and p.P1082S, none of the missense variants were listed in these databases. The Allele frequencies of the missense variants were searched from the dbSNP (28) and 1000 genomes (http://www.1000genomes.org/) databases. None of the variants were reported in either of these databases, nor were they polymorphism with a population-based allele frequency $>0.01$ (Table II), except for c.3244C $>$ T, which had an allele frequency of 0.0014 .

The distribution of the truncating mutations and missense variants are shown in Fig. 1. The majority of the mutations were located in the MutS domain, but no spatial preference of the mutation site in the domain was found. The molecular features of the tumors are shown in Table II and the clinical features of the carriers are shown in Table III.

Molecular features of the variant carriers. As shown in Table II, the dinucleotide markers were more stable than the mononucleotide markers in tumors from the pathogenic variant carriers, but not in the missense variant carriers. Of the nine tumors from the carriers of the pathogenic variants, eight showed the absence of staining for MSH6 and retained staining for both MLH1 and PMS2 in the IHC analysis. Only one tumor from $\mathrm{pR} 1321 \mathrm{X}$ mutation carrier showed expression of all proteins. In short, in seven of the nine tumors from the pathogenic variant carriers, IHC showed a specific pattern with negative staining for MSH6 and positive staining for MSH2, MLH1 and PMS2. On the other hand, four of the eight tumors from the missense variant carriers showed a loss of MSH6 expression and the other four showed a loss of the expression of both MLH1 and PMS2. Of the four tumors showing negative staining for MSH6, three had also lost the expression of the MSH2 protein. Most tumors without MLH1 expression had the BRAF mutation and/or hypermethylation of $M L H 1$ promoter (Table II). 


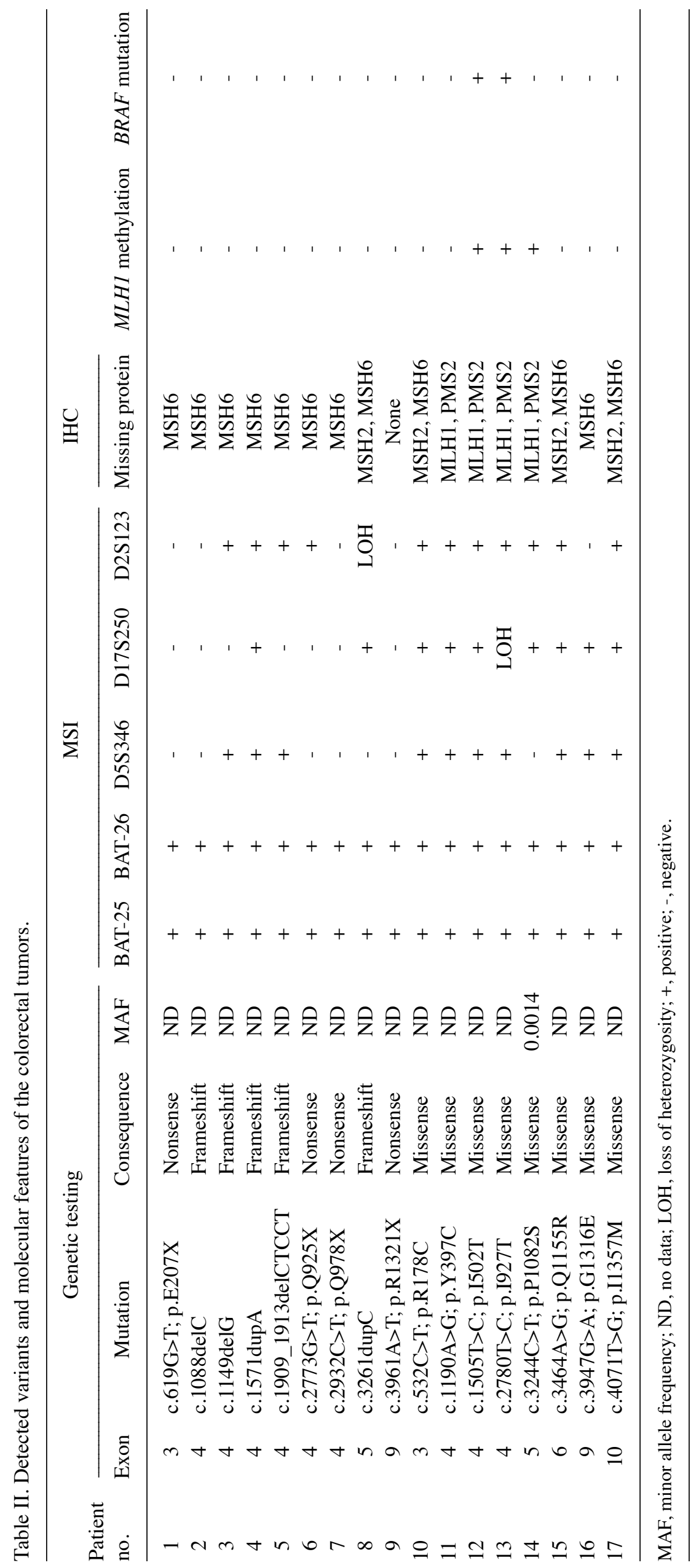




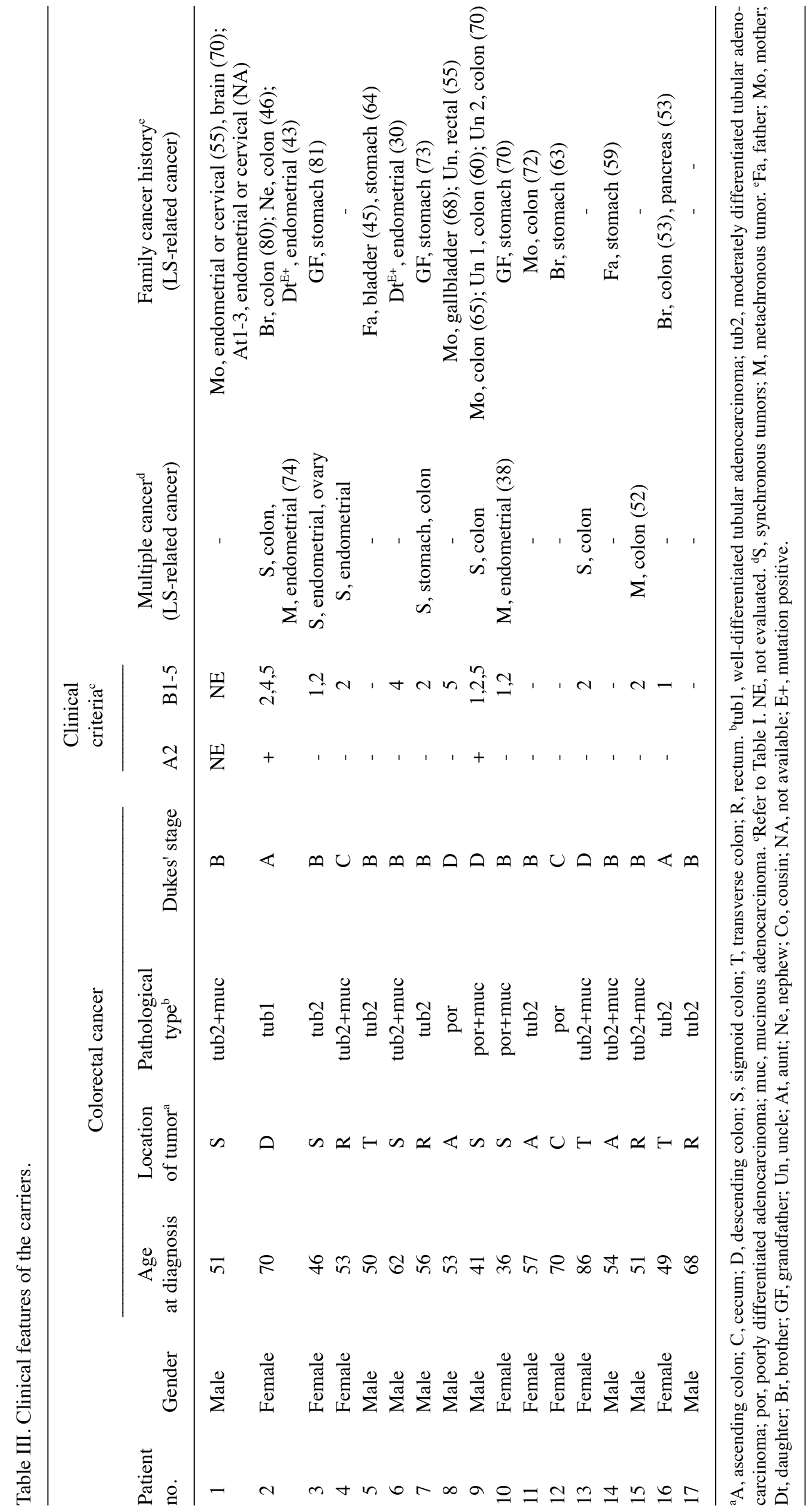


Table IV. Prediction results of in silico tools in missense variants.

\begin{tabular}{|c|c|c|c|c|c|}
\hline \multirow[b]{2}{*}{ Missense variant } & \multirow[b]{2}{*}{$\mathrm{CoDP}^{\mathrm{a}}$} & \multirow[b]{2}{*}{ SIFT $^{b}$} & \multicolumn{2}{|c|}{ PolyPhen- $2^{c}$} & \multirow[b]{2}{*}{ PON-MMR $^{\mathrm{d}}$} \\
\hline & & & HumDiv & HumVar & \\
\hline $\mathrm{R} 178 \mathrm{C}$ & $0(0.009)$ & 0 & 1 & 0 & $\mathrm{U}$ \\
\hline Y397C & $2(0.976)$ & 1 & 2 & 1 & $\mathrm{U}$ \\
\hline $\mathrm{I} 502 \mathrm{~T}$ & $0(0.001)$ & 0 & 0 & 0 & 0 \\
\hline I927T & $0(0.113)$ & 1 & 2 & 1 & $\mathrm{U}$ \\
\hline P1082S & $0(0.018)$ & 0 & 0 & 0 & $\mathrm{U}$ \\
\hline Q1155R & $2(0.980)$ & 1 & 2 & 2 & $\mathrm{U}$ \\
\hline G1316E & $2(0.999)$ & 1 & 2 & 2 & $\mathrm{U}$ \\
\hline I1357M & $0(0.002)$ & 1 & 0 & 0 & 0 \\
\hline
\end{tabular}

${ }^{a}$ CoDP: 0, minor impact on molecular function; 1 , moderate impact; 2 , likely impair molecular function. The number in parentheses shows the prediction score. ${ }^{b}$ SIFT: 0 , tolerated; 1 , not tolerated. ${ }^{~}$ PolyPhen-2: 0 , benign; 1 , possibly damaging; 2 , probably damaging. ${ }^{\mathrm{d}}$ PON-MMR: 0 , neutral; $\mathrm{U}$, unclassified variant.

Clinical features of the variant carriers. The mean and median age of the patients carrying pathogenic variants at the diagnosis of CRC was 53.6 and 53.0 years (range, 41-70, patient nos. 1-9), respectively. The mean and median age of the patients with hypermethylation in the $M L H 1$ promoter (patient nos. 12-14) were 70.0 and 70.0 years (range, 54-86), while those of the patients without hypermethylation was 53.0 and 54.0 years (range, 36-68) in the missense variant carriers (patient nos. 10,11,15-17), respectively (Table II). The patients with hypermethylation in the $M L H 1$ promoter tended to develop CRC at a more advanced age than did the carriers of the pathogenic variants (Student's t-test, $\mathrm{P}=0.040$; Mann-Whitney U-test, $\mathrm{P}=0.064)$. Meanwhile, in the pathogenic variant carriers, including affected relatives, the mean and median ages at the diagnosis of endometrial cancer was 49.2 and 46.0 years (range, 30-74) respectively, indicating that endometrial cancer developed earlier than CRC.

As shown in Table III, of the nine pathogenic variant carriers, seven had tumors in the left side colon or rectum, and five had multiple cancers. In the histological findings of the CRCs, four pathogenic variant cases contained mucinous adenocarcinoma and poorly differentiated adenocarcinoma was observed in two cases. Tumor infiltrating lymphocytes were observed in most cases (data not shown). Concerning the clinical criteria, of the nine pathogenic variant carriers, two fulfilled the Amsterdam criteria II and seven fulfilled one of the criteria included in the revised Bethesda guidelines. Four cases had a family cancer history that more than two relatives affected LS-related cancer. On the other hand, in the missense variant carriers, none of the patients fulfilled the Amsterdam criteria II. Although four fulfilled at least one of the criteria in the revised Bethesda guidelines, the B4 and B5 criteria related to a family history of LS-related cancer were not fulfilled in any of the cases.

Prediction by in silico tools. We also investigated the impact of the missense variants of MSH6 to classify them as either pathogenic or non-pathogenic by an in silico prediction using the CoDP, SIFT, PolyPhen-2 and PON-MMR programs
(Table IV). PON-MMR classified six variants as unclassified variants. Excluding the results obtained from the PON-MMR program, five missense variants gave consistent results; the Y397C, Q1155R and G1316E variants were predicted to be pathogenic, and the I502T and P1082S variants were predicted to be non-pathogenic.

\section{Discussion}

Accumulating evidence indicates that difference of the mutated MMR genes, geographic region and gender affects the clinical phenotype of Lynch syndrome (29). However, the majority of previous studies have been conducted on Caucasian European populations and there have been few reports on Japanese LS patients carrying MSH6 mutations. In the present study, we screened colorectal cancer by MSI testing, we performed a germline mutation analysis and identified nine pathogenic and eight unclassified missense variants in MSH6, 15 of which were novel variants. A comprehensive investigation of the molecular and clinical characteristics of the carriers of those variants was performed, and several unique features in Japanese LS patients carrying MSH6 mutations were found.

The mean age at the diagnosis of colorectal carcinoma in the MSH6 pathogenic variant carriers was 53.6 years, and the earliest onset was at age 41 in the present study. It was previously reported that the mean age at diagnosis of colorectal carcinoma in $\mathrm{MLH1}$ or $\mathrm{MSH} 2$ mutation carriers was 43-46 years but was 51-57 years in MSH6 mutation carriers in Western countries (30). Thus, the development of colorectal carcinomas in Japanese MSH6 mutation carriers was similar to the previous reports from Western countries. On the other hand, mean age at the diagnosis of endometrial carcinoma was 49.2 years, and the earliest onset was at age 30 (the daughter of patient no. 6) in the present study. Previous studies from Western countries reported that mean age at the diagnosis of endometrial cancer was $\sim 48$ years in $M L H 1$ or $M S H 2$ mutation carriers $(9,30,31)$ and 56.5 years in $M S H 6$ mutation carriers (32). Thus, the endometrial carcinoma in MSH6 mutation carriers was generally diagnosed at an older age ( $\sim 5$ years later) 
than colorectal carcinoma in the previous reports. However, Japanese MSH6 mutation carriers tended to develop endometrial carcinoma earlier than colorectal carcinoma. Although the small number of cancer cases limited statistical power, this result may provide evidence to support the development of effective surveillance programs for Japanese LS due to MSH6 mutations. In other words, the finding of the early-onset of endometrial carcinoma in MSH6 mutation carriers in our study suggests that the surveillance program for endometrial carcinoma may need to be started from an earlier age in Japan.

Another marked finding of the present study was the location of colorectal carcinomas in MSH6 mutation carriers. The CRCs from MMR mutation carriers are mostly located in the right-sided colon, in contrast to sporadic colorectal carcinomas, which are generally detected in the distal region (17). However, the present study noted that the majority $(77.8 \%)$ of colorectal carcinomas were located distally in Japanese MSH6 mutation carriers, and these were all MSI-H. Berends et al (8) reported that two-thirds of the colorectal carcinomas from MSH6 mutation carriers were located on the left side, but more than half of these were MSS or MSI-low. Furthermore, approximately one-third of the tumors were detected to express the MSH6 protein in their study, but only one tumor (11\%) was detected with MSH6 protein expression by IHC in our study. Thus, the MSI and IHC status differed in the two studies.

In general, the Amsterdam criteria and Bethesda guidelines are used to screen patients for Lynch syndrome. Of the nine mutation carriers in the present study, two fulfilled the Amsterdam criteria II and seven fulfilled the revised Bethesda guidelines but these criteria missed one LS case. Therefore, MSI testing for all CRC cases would be a useful screening method for high-risk individuals with an MSH6 mutation. However, some MSH6 mutation carriers developed MSI-L or MSS tumors (32). While, an IHC analysis is another potential screening strategy for LS, a recent study reported that $\sim 30 \%$ of colon tumors in carriers of pathogenic MSH6 mutations still showed MSH6 protein expression (33). Thus, neither of these methods is a perfect screening tool, and a combination of MSI and IHC testing to screen MSH6 mutation carriers would be a more appropriate method.

To classify the missense variants detected in the present study, we integrated the molecular and clinical data and the results of an in silico prediction. A classification system for VUS was previously proposed, based on the probability of pathogenicity (34). The system classifies genetic variants into five categories: class-5, definitely pathogenic; class-4; likely pathogenic; class-3, uncertain; class-2, likely not pathogenic or of little clinical significance; and class-1, definitely neutral. Based on this system, the eight missense variants were classified into five categories.

Hypermethylation of the $M L H 1$ promoter and loss of MLH1 and PMS2 expression detected by IHC was observed in the tumors from the I502T, I927T and P1082S variant carriers. Two of these patients were over 70 years old at the time of the diagnosis of CRC, and two did not fulfill the clinical criteria. Therefore, these tumors were considered to be sporadic and these variants were categorized as class- 2 or -3 .

The R178C variant carrier also harbored an $\mathrm{H} 639 \mathrm{Y}$ variant in $M S H 2$. The variant in $M S H 2$ was classified as pathogenic by integrative consideration of a clinical report (35), functional assay results (35-37) and in silico prediction. The loss of MSH2 and MSH6 expression was also observed by IHC and R178C was predicted to be non-pathogenic by the CoDP, SIFT and PolyPhen-2 programs. However, since the R178C (c.532C>T) variant has thus far not been reported, it is considered to be class-3.

The remaining four variants, i.e. Y397C, Q1155R, G1316E and I1357T, did not show either hypermethylation of the MLH1 promoter or the BRAF mutation. The Q1155R carrier had multiple CRCs and the G1316E carrier was diagnosed with CRC before age 50. In addition, the tumors of these variant carriers had lost the MSH6 protein, as determined by IHC and demonstrated by MSI-H. We also performed an in silico prediction, and most tools predicted that Q1155R and G1316E were pathogenic. After taking all these findings into account, these variants were considered to be class-4.

In a previous report, the Y397C mutation was found in a patient with ureter cancer, which is one of the LS-associated cancers (38), but the tumor did not have MSI. The in silico analysis predicted that it was pathogenic. Since the information was limited, this variant is presently considered to be class-3.

The carrier of I1357T did not fulfill any of the clinical criteria, and this variant was predicted to be non-pathogenic by the in silico tools. However, the rectal carcinoma of this carrier showed MSI-H and loss of the MSH6 protein. Therefore, I1357T is considered to be class-3.

The category of all variants can be altered by the inclusion of additional data, such as the results of a segregation analysis and functional assays of the MMR activity. More MSH6 variant carriers will need to be identified and analyzed and the classification of the variants will be continuously refined based on the accumulation of clinical and molecular data.

\section{Acknowledgements}

We thank Akemi Takahashi for the technical assistance. The present study was supported in part by a grant-in-aid for Cancer Research from the Ministry of Health, Labour and Welfare.

\section{References}

1. Lynch HT and de la Chapelle A: Hereditary colorectal cancer. N Engl J Med 348: 919-932, 2003.

2. Grover S and Syngal S: Genetic testing in gastroenterology: Lynch syndrome. Best Pract Res Clin Gastroenterol 23: 185-196, 2009.

3. Hampel H, Frankel WL, Martin E, et al: Feasibility of screening for Lynch syndrome among patients with colorectal cancer. J Clin Oncol 26: 5783-5788, 2008.

4. Aaltonen LA, Salovaara R, Kristo P, et al: Incidence of hereditary nonpolyposis colorectal cancer and the feasibility of molecular screening for the disease. N Engl J Med 338: 1481-1487, 1998.

5. Wijnen J, de Leeuw W, Vasen H, et al: Familial endometrial cancer in female carriers of MSH6 germline mutations. Nat Genet 23: 142-144, 1999.

6. Vasen HF, Mecklin JP, Khan PM and Lynch HT: The International Collaborative Group on Hereditary Non-Polyposis Colorectal Cancer (ICG-HNPCC). Dis Colon Rectum 34: 424-425, 1991.

7. Baglietto L, Lindor NM, Dowty JG, et al: Risks of Lynch syndrome cancers for MSH6 mutation carriers. J Natl Cancer Inst 102: 193-201, 2010.

8. Berends MJW, Wu Y, Sijmons RH, et al: Molecular and clinical characteristics of $M S H 6$ variants: an analysis of 25 index carriers of a germline variant. Am J Hum Genet 70: 26-37, 2002.

9. Hendriks YMC, Wagner A, Morreau H, et al: Cancer risk in hereditary nonpolyposis colorectal cancer due to MSH6 mutations: impact on counseling and surveillance. Gastroenterology 127: $17-25,2004$. 
10. Wijnen JT, Vasen HF, Khan PM, et al: Clinical findings with implications for genetic testing in families with clustering of colorectal cancer. N Engl J Med 339: 511-518, 1998.

11. Cunningham JM, Christensen ER, Tester DJ, et al: Hypermethylation of the $h M L H 1$ promoter in colon cancer with microsatellite instability. Cancer Res 58: 3455-3460, 1998.

12. Herman JG, Umar A, Polyak K, et al: Incidence and functional consequences of $h M L H 1$ promoter hypermethylation in colorectal carcinoma. Proc Natl Acad Sci USA 95: 6870-6875, 1998.

13. Kane MF, Loda M, Gaida GM, et al: Methylation of the $h M L H 1$ promoter correlates with lack of expression of hMLH1 in sporadic colon tumors and mismatch repair-defective human tumor cell lines. Cancer Res 57: 808-811, 1997.

14. Bessa X, Ballesté B, Andreu M, et al: A prospective, multicenter, population-based study of $B R A F$ mutational analysis for Lynch syndrome screening. Clin Gastroenterol Hepatol 6: 206-214, 2008.

15. Akagi K, Uchibori R, Yamaguchi K, Kurosawa K, Tanaka Y and Kozu T: Characterization of a novel oncogenic $K$-ras mutation in colon cancer. Biochem Biophys Res Commun 352: 728-732, 2007.

16. Vasen HF, Watson P, Mecklin JP and Lynch HT: New clinical criteria for hereditary nonpolyposis colorectal cancer (HNPCC, Lynch syndrome) proposed by the International Collaborative group on HNPCC. Gastroenterology 116: 1453-1456, 1999.

17. Umar A, Boland CR, Terdiman JP, et al: Revised Bethesda Guidelines for hereditary nonpolyposis colorectal cancer (Lynch syndrome) and microsatellite instability. J Natl Cancer Inst 96: 261-268, 2004

18. Ohsawa T, Sahara T, Muramatsu S, et al: Colorectal cancer susceptibility associated with the $h M L H 1$ V384D variant. Mol Med Rep 2: 887-891, 2009.

19. Asaka S, Arai Y, Nishimura Y, et al: Microsatellite instabilitylow colorectal cancer acquires a KRAS mutation during the progression from Dukes' A to Dukes' B. Carcinogenesis 30: 494-499, 2009

20. Campbell PT, Curtin K, Ulrich CM, et al: Mismatch repair polymorphisms and risk of colon cancer, tumour microsatellite instability and interactions with lifestyle factors. Gut 58: 661-667, 2009.

21. Jass JR: Classification of colorectal cancer based on correlation of clinical, morphological and molecular features. Histopathology 50: 113-130, 2007.

22. Terui H, Akagi K, Kawame $\mathrm{H}$ and Yura K: CoDP: predicting the impact of unclassified genetic variants in MSH6 by the combination of different properties of the protein. J Biomed Sci 20: 25 , 2013.

23. Ng PC and Henikoff S: Predicting deleterious amino acid substitutions. Genome Res 11: 863-874, 2001

24. Adzhubei IA, Schmidt S, Peshkin L, et al: A method and server for predicting damaging missense mutations. Nat Methods 7 : $248-249,2010$
25. Ali H, Olatubosun A and Vihinen M: Classification of mismatch repair gene missense variants with PON-MMR. Hum Mutat 33: 642-650, 2012.

26. Woods MO, Williams P, Careen A, Edwards L, Bartlett S, McLaughlin JR and Younghusband HB: A new variant database for mismatch repair genes associated with Lynch syndrome. Hum Mutat 28: 669-673, 2007.

27. Stenson PD, Ball E V, Mort M, Phillips AD, Shaw K and Cooper DN: The Human Gene Mutation Database (HGMD) and its exploitation in the fields of personalized genomics and molecular evolution. Curr Protoc Bioinformatics 39: 1.13, 2012.

28. Sherry ST, Ward MH, Kholodov M, Baker J, Phan L, Smigielski EM and Sirotkin K: dbSNP: the NCBI database of genetic variation. Nucleic Acids Res 29: 308-311, 2001.

29. Engel C, Loeffler M, Steinke V, et al: Risks of less common cancers in proven mutation carriers with lynch syndrome. J Clin Oncol 30: 4409-4415, 2012.

30. Peltomäki P: Lynch syndrome genes. Fam Cancer 4: 227-232, 2005.

31. Vasen HFA: Review article: The Lynch syndrome (hereditary nonpolyposis colorectal cancer). Aliment Pharmacol Ther 26: 113-126, 2007.

32. Zhao Y, Hu F, Wang F, Han B, Li D, Li X and Zhu S: Metaanalysis of MSH6 gene mutation frequency in colorectal and endometrial cancers. J Toxicol Environ Health A 72: 690-697, 2009.

33. Okkels H, Lindorff-Larsen K, Thorlasius-Ussing O, et al: MSH6 mutations are frequent in hereditary nonpolyposis colorectal cancer families with normal pMSH6 expression as detected by immunohistochemistry. Appl Immunohistochem Mol Morphol 20: 470-477, 2012.

34. Plon SE, Eccles DM, Easton D, et al: Sequence variant classification and reporting: recommendations for improving the interpretation of cancer susceptibility genetic test results. Hum Mutat 29: 1282-1291, 2008

35. Ellison AR, Lofing J and Bitter GA: Functional analysis of human MLH1 and MSH2 missense variants and hybrid humanyeast MLH1 proteins in Saccharomyces cerevisiae. Hum Mol Genet 10: 1889-1900, 2001.

36. Gammie AE, Erdeniz N, Beaver J, Devlin B, Nanji A and Rose MD: Functional characterization of pathogenic human MSH2 missense mutations in Saccharomyces cerevisiae. Genetics 177: 707-721, 2007.

37. Polaczek P,Putzke AP, Leong K and Bitter GA: Functional genetic tests of DNA mismatch repair protein activity in Saccharomyces cerevisiae. Gene 213: 159-167, 1998.

38. Furihata M, Shuin T, Takeuchi T, Sonobe H, Ohtsuki Y, Akiyama Y and Yuasa Y: Missense mutation of the hMSH6 and p53 genes in sporadic urothelial transitional cell carcinoma. Int J Oncol 16: 491-496, 2000 